\title{
MULTIPLE CHOICE QUESTION - PHARMACOTHERAPY OF EPILEPSY
}

Q.1 Which of the following Anti-Epileptic Drugs (AEDs) should be avoided in an ICU patient who is on meropenem for pneumonia?
A) Sodium Valproate
B) Levetiracetam
C) Lacosamide
D) Midazolam

Q.2 All of the following AEDs except one are consider to be enzyme-inducing AEDs
A) Phenytoin
B) Carbamazepine
C) Primidone
D) Sodium Valproate

Q.3 The proposed mechanism of action for Levetiracetam is:
A) Modifies synaptic release of glutamate and GABA through action on vesicular function
B) Enhances slow inactivation of voltage-gated Sodium Channels
C) Inhibits Carbonic Anhydrase
D) Irreversibly inhibits GABA aminotransferase

Q.4 Rufinamide is indicated for
A) Adjunctive treatment of focal epilepsy
B) Adjunctive treatment of the Lennox-Gastaut Syndrome
C) Generalized tonic-clonic seizures
D) Status Epilepticus

Q.5 Which of the following side-effects can be attributed to Topiramate?
A) Nephrolithiasis
B) Gingival hyperplasia
C) Hemorrhagic Pancreatitis
D) Weight gain

Q.6 Fatal cases of aplastic anemia have been reported with use of the following AEDs:
A) Levetiracetam and Pregablin
B) Carbamazepine and Felbamate
C) Lacosamide and tiagabine
D) Phenytoin and phenobarbital

Q.7 Ethosuximide is indicated for treatment of:
A) Focal epilepsy
B) Generalized tonic-clonic seizures
C) Generalized non-motor (absence) seizures
D) Unclassified epilepsy 
Q.8 Idiosyncratic reactions of Valproate include all of the following except
A) Hepatotoxicity
B) Pancreatitis
C) Thrombocytopenia
D) Psychosis

Q.9 Chronic dose-related adverse effects of valproate include all of the following except
A) Weight gain
B) Hair loss
C) Peripheral neuropathy
D) Endocrine and metabolic changes

Q.10 Vigabitrin is indicated for myoclonic epilepsy
A) True
B) False

Dr. Sunita Dergalust PharmD, BCPS Adjunct Clinical Faculty at USC School of Pharmacy Clinical Pharmacist in Neurology and Neurosurgery Residency Program Director: PGY2 Neurology Specialty Pharmacy Program West LA VA Medical Center VA Greater Los Angeles Healthcare System 11301 Wilshire Blvd (119) Los Angeles, CA 90073

\section{Answer Keys -}

1) $\mathrm{A}$

2) $\mathrm{D}$

3) $\mathrm{A}$

4) $\mathrm{B}$

5) $\mathrm{A}$

6) $B$

7) $\mathrm{C}$

8) $\mathrm{D}$

9) $\mathrm{C}$

10) $B$ 